\title{
Lymph node fibrosis: a structural barrier to unleashing effective vaccine immunity
}

\author{
Boris Julg and Galit Alter \\ Ragon Institute of MGH, MIT and Harvard, Cambridge, Massachusetts, USA. Infectious Disease Unit, Massachusetts General Hospital, Boston, Massachusetts, USA.
}

\begin{abstract}
There is marked variability in vaccine efficacy among global populations. In particular, individuals in low- to middle-income countries have been shown to be less responsive to vaccines than those from developed nations. Several factors, including endemic infections, nutrition, genetics, and gut microbiome composition, have been proposed to underlie discrepancies in vaccine response. In this issue of the $J C l$, Kityo et al. evaluated response to yellow fever virus vaccine, inflammation, and lymphatic tissue architecture and fibrosis in three cohorts: two from the U.S. and one from Uganda. Compared with the U.S. subjects, the Ugandan cohort exhibited enhanced cytokine responses, increased lymph node fibrosis, reduced $\mathrm{CD4}{ }^{+} \mathrm{T}$ cell levels, and reduced vaccine response. Together, these results provide a link among chronic inflammation, damaged lymphoid architecture, and poor vaccine outcome, and set the stage for future studies to identify strategies to overcome these barriers.
\end{abstract}

\section{A geographic map of vaccine failure}

Vaccination against infectious organisms has proven to be one of the most effective public health strategies for controlling and preventing disease. Vaccination strategies have shown specific benefit in low- and middle-income countries, where recent estimates suggest that by 2030 up to 36 million deaths will be averted simply by immunization against 10 common infections (1). Despite the dramatic successes predicted by models, accumulating evidence suggests that vaccine responses vary geographically and that efficacy rates, particularly for oral vaccines against polio, cholera, and rotavirus, are reduced in low- and middle-income countries (2). For example, efficacy rates of Rotarixä, a vaccine against severe rotavirus-induced gastroenteritis, have been reported to be less than $50 \%$ for infants vaccinated in Malawi compared with their European counterparts (3). Similarly, deficits in immunogenicity and/or efficacy have been documented for live-attenuated oral cholera vaccines (4) and for the trivalent oral poliovirus vaccine (TOPV), with up to a $30 \%$ reduction in seroconversion rates following administration in children in lowincome countries compared with children in industrialized countries (5). It has even been suggested that the reduced OPV efficacy in low-income countries has contributed to the delayed global eradication of polio virus.

Interestingly, geographic variation in vaccine responsiveness is not limited to oral vaccines in infants but was described two decades ago for the Bacillus CalmetteGuerin (BCG) vaccine against mycobacterium tuberculosis (TB) (6). BCG immunogenicity has been strongly linked to geographic latitude, with diminished efficacy closer to the equator (7). Similar geographic variation in vaccine immunogenicity was also observed for the yellow fever virus vaccine (YFV), with lower levels of neutralizing antibodies described in an adult Ugandan population compared

Related Article: p. 2763

Conflict of interest: The authors have declared that no conflict of interest exists.

Reference information: / Clin Invest. 2018;128(7):2743-2745. https://doi.org/10.1172/JCI121053. 
deficiencies in critical nutrients and the frequent occurrence of enteric dysfunction, the composition of the gut bacterial microbiota itself has been associated with vaccine immunogenicity (11). Significant geographic differences have been linked to microbiome composition (20), and recent data highlight similarities in microbiome composition in rotavirus vaccine responders in Ghana and the Netherlands compared with Ghanaian infant nonresponders (21). Similarly, the specific presence of particular bacterial species in the stool microbiota of Bangladeshi infants, for example, has been associated with enhanced vaccine-specific IgG and $\mathrm{T}$ cell proliferative responses to the oral polio, BCG, tetanus toxoid, and HBV vaccines (22). However, results implicating the microbiome in vaccine response variability have thus far been purely associative, and causal links to the microbiota, or information on how it may be exploited to improve immunity, have yet to emerge.

\section{Coinfections: vaccine antagonists}

Chronic exposure to and recurrent infections with intestinal helminths, Plasmodium, Mycobacterium, and other endemic pathogens affect more than a third of the global population, with a clear predominance in low-income countries where infection with at least one persistent pathogen is frequently found. Reduced responses to vaccinations for tetanus toxoid (23), cholera (24), and BCG (25) have been associated with helminth coinfection, and children with malaria have been shown to mount weaker antibody responses to tetanus toxoid, Haemophilus influenzae, meningococcal polysaccharide, and Salmonella typhi vaccination (26) than uninfected children. While some parasitic infections do not affect the initial generation or levels of protective antibody, parasitic infections clearly affect the durability of these immune responses, as reported for HBV or tetanus toxoid vaccines in chronic schistosomiasis (27). Several mechanisms have been proposed for the antagonizing effects that coinfection has on vaccine durability. These include skewing of lymphocyte effector functions, induction of antiinflammatory cytokines, altered antigen presenting cell function, and reduced antigen processing and presentation, all of which result in poor or altered priming of
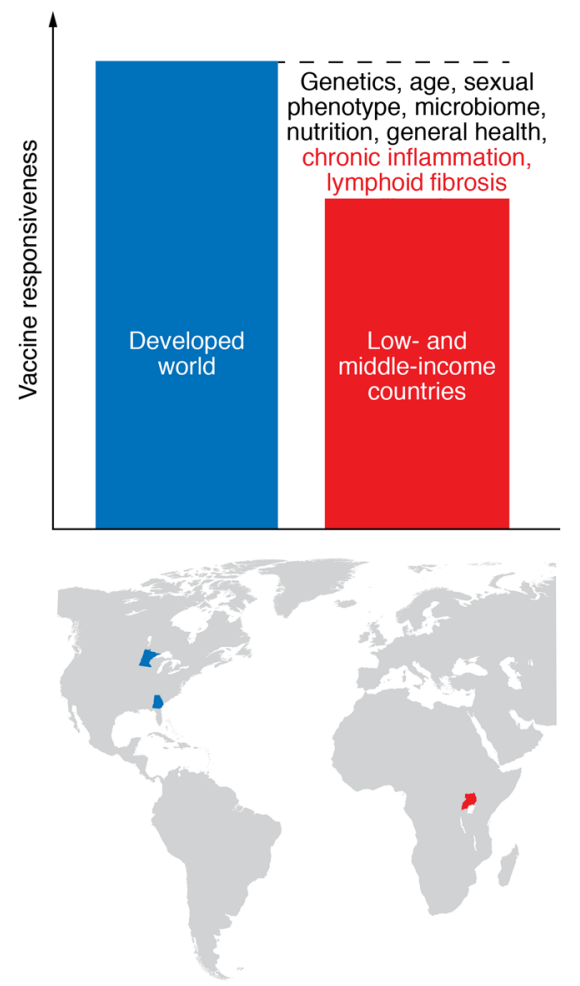

Figure 1. Factors driving lower vaccine responsiveness around the globe. The cartoon bar graph depicts the observed differences in vaccine responsiveness around the globe, and highlights factors that have been associated with vaccine immunogenicity. Factors listed in black have been previously published. Factors listed in red represent the findings in the manuscript by Kityo et al. which evaluated differences in vaccine response in subjects from the U.S. (Minnesota and Georgia, blue) and subjects from Uganda (red).

adaptive immune responses (reviewed in ref. 28). Moreover, chronic infection and associated inflammation have been associated with altered lymphoid tissue architecture, particularly in HIV infection, and linked to T cell depletion (29). However, the exact consequences of such changes in the context of vaccination have remained poorly understood, until now.

\section{Structural effects on vaccine- induced immunity}

In this issue, Kityo et al. (30) specifically explore the role of inflammation, tissue architecture, and fibrosis in modulating vaccine-induced immunity. In a tour de force, the study, which includes lymph node (LN) excisions and immune profiling, specifically aimed to define the linkage among LN inflammation, fibrosis, chronic immune activation, and vaccine- induced immunity. The authors hypothesized that similar to HIV-induced changes in lymphoid architecture (29), recurrent coinfections may contribute to the reduced vaccine responsiveness in low-income countries by altering immune activationinduced lymphoid architecture. Kityo and colleagues (30) recruited three different cohorts to address their hypothesis, specifically $30 \mathrm{HIV}^{-}$Ugandans, a subset of which was subsequently given YFV; a group of $\mathrm{HIV}^{-}$participants from Atlanta, USA, who received YFV; and a group of $10 \mathrm{HIV}^{-}$individuals from Minnesota, USA. LNs were collected from the Ugandans and the Minnesotans. Ugandans exhibited enhanced inflammatory cytokine responses, marked by elevated levels of TGF- $\beta$, IL-6, IL-4, IL-21, and MIP-1 $\beta$, compared with subjects from the U.S., suggesting distinct inflammatory profiles across geographic regions of the globe. Additionally, collagen levels were markedly increased in the $\mathrm{T}$ cell zone (TZ) in LNs of Ugandans compared with those from U.S. controls, similar to what has been previously reported in $\mathrm{HIV}^{+}$ individuals (29). Furthermore, there was a significant inverse correlation between $\mathrm{TZ}$ collagen deposition and $\mathrm{TZ} \mathrm{CD}^{+} \mathrm{T}$ cell frequencies, known to be essential drivers of effective vaccine-induced antibody responses, suggesting $\mathrm{T}$ cell depletion in the Ugandans, even in the absence of HIV infection. To further determine if the observed differences in lymphatic tissue anatomy were associated with vaccine responsiveness, Kityo and colleagues (30) examined draining LNs 10-14 days after YFV and measured plasma YFV antibody titers by plaque reduction assay. Interestingly, not only was there a relative lack of primary follicles with fewer secondary follicles (germinal centers [GCs]) prior to vaccination in the Ugandan cohort, but also the majority of these individuals did not have a detectable increase in primary or secondary follicle formation following vaccination. Moreover, these immunological defects were consistent with a poor antibody response in the Ugandan cohort and correlated with increased damage in the fibroblastic reticular cell network (FRCn), an anatomic correlate of poor vaccine response. Thus, these data provide a definitive link among chronic inflammation, damaged lymphoid architecture, and poor vaccine outcome (Figure 1). 


\section{Summary and future directions}

Vaccine immunogenicity is frequently reduced in vulnerable populations, including those living in low-income countries, the elderly, and other marginalized populations (e.g., drug users) in the developed world. A common thread among many of these populations is the marked elevation and/or alteration of inflammation. The results presented by Kityo et al. (30) now propose a mechanism that may transcend across global populations and may provide novel insights into dampened vaccine immunity, with particular focus on the role of inflammation in shaping LN anatomy. Specifically, increased inflammation is associated with fibrotic damage to the $\mathrm{TZ}$, resulting in dampened ability for GC generation and priming of vaccinespecific antibodies. The insights of Kityo et al. provide critical clues to the vaccine field and identify a target obstacle that must be overcome to attain comparable immunity at a global level. While attempts to reduce or reverse LN fibrosis in $\mathrm{HIV}^{+}$individuals with antifibrotic agents like the angiotensin receptor blocker and PPAR- $\gamma$ agonist telmisartan have been unsuccessful (31), modified vaccine regimens, novel adjuvants, as well as next-generation vaccine delivery strategies may counter the limitations of fibrotic lymph tissues. Thus, the study by Kityo et al. represents a significant step in defining the immune system's Achilles' heel and is a critical first step in achieving global immunity to prevent the devastation of infectious diseases.

\section{Acknowledgments}

This work was supported by NIH K08 AI 106408 (BJ).

Address correspondence to: Galit Alter, Ragon Institute of MGH, MIT and Harvard, 400 Technology Square, Cambridge, Massachusetts 02139, USA. Phone: 617.724.0546; Email:galter@mgh.harvard.edu.
1. Chang AY, et al. The equity impact vaccines may have on averting deaths and medical impoverishment in developing countries. Health Aff (Millwood). 2018;37(2):316-324.

2. Cherian T, Wang S, Mantel C. Rotavirus vaccines in developing countries: the potential impact, implementation challenges, and remaining questions. Vaccine. 2012;30 Suppl 1:A3-A6.

3. Madhi SA, et al. Effect of human rotavirus vaccine on severe diarrhea in African infants. $N$ Engl JMed. 2010;362(4):289-298.

4. Suharyono, et al. Safety and immunogenicity of single-dose live oral cholera vaccine CVD 103-HgR in 5-9-year-old Indonesian children. Lancet. 1992;340(8821):689-694.

5. Patriarca PA, Wright PF, John TJ. Factors affecting the immunogenicity of oral poliovirus vaccine in developing countries: review. Rev Infect Dis. 1991;13(5):926-939.

6. Fine PE. Variation in protection by BCG: implications of and for heterologous immunity. Lancet. 1995;346(8986):1339-1345.

7. Black GF, et al. BCG-induced increase in interferon-gamma response to mycobacterial antigens and efficacy of BCG vaccination in Malawi and the UK: two randomised controlled studies. Lancet. 2002;359(9315):1393-1401.

8. Muyanja E, et al. Immune activation alters cellular and humoral responses to yellow fever 17D vaccine. JClin Invest. 2014;124(7):3147-3158.

9. Mentzer AJ, O'Connor D, Pollard AJ, Hill AV. Searching for the human genetic factors standing in the way of universally effective vaccines. Philos Trans R Soc Lond, B, Biol Sci. 2015;370(1671):20140341.

10. Flanagan KL, Fink AL, Plebanski M, Klein SL. Sex and gender differences in the outcomes of vaccination over the life course. Annu Rev Cell Dev Biol. 2017;33:577-599.

11. Lynn DJ, Pulendran B. The potential of the microbiota to influence vaccine responses. J Leukoc Biol. 2018;103(2):225-231.

12. Yucesoy B, et al. Genetic variants within the $\mathrm{MHC}$ region are associated with immune responsiveness to childhood vaccinations. $\mathrm{Vac}$ cine. 2013;31(46):5381-5391.

13. Ovsyannikova IG, Dhiman N, Jacobson RM, Poland GA. Human leukocyte antigen polymorphisms: variable humoral immune responses to viral vaccines. Expert Rev Vaccines. 2006;5(1):33-43.

14. Yucesoy B, et al. Influence of cytokine gene variations on immunization to childhood vaccines. Vaccine. 2009;27(50):6991-6997.

15. Höhler T, et al. Differential genetic determination of immune responsiveness to hepatitis B surface antigen and to hepatitis A virus: a vaccination study in twins. Lancet. 2002;360(9338):991-995.

16. Tan PL, Jacobson RM, Poland GA, Jacobsen SJ, Pankratz VS. Twin studies of immunogenicity - determining the genetic contribution to vaccine failure. Vaccine. 2001;19(17-19):2434-2439.

17. Newport MJ, et al. Genetic regulation of immune responses to vaccines in early life. Genes Immun. 2004;5(2):122-129.

18. Powell GM. Response to live attenuated measles vaccine in children with severe kwashiorkor. Ann Trop Paediatr. 1982;2(3):143-145.

19. Brüssow H, Sidoti J, Dirren H, Freire WB. Effect of malnutrition in Ecuadorian children on titers of serum antibodies to various microbial antigens. Clin Diagn Lab Immunol. 1995;2(1):62-68.

20. Yatsunenko T, et al. Human gut microbiome viewed across age and geography. Nature. 2012;486(7402):222-227.

21. Harris VC, et al. Significant correlation between the infant gut microbiome and rotavirus vaccine response in rural Ghana. J Infect Dis. 2017;215(1):34-41.

22. Huda MN, et al. Stool microbiota and vaccine responses of infants. Pediatrics. 2014;134(2):e362-e372.

23. Nookala S, Srinivasan S, Kaliraj P, Narayanan RB, Nutman TB. Impairment of tetanus-specific cellular and humoral responses following tetanus vaccination in human lymphatic filariasis. Infect Immun. 2004;72(5):2598-2604.

24. Cooper PJ, et al. Human infection with Ascaris lumbricoides is associated with suppression of the interleukin-2 response to recombinant cholera toxin B subunit following vaccination with the live oral cholera vaccine CVD 103-HgR. Infect Immun . 2001;69(3):1574-1580.

25. Elias D, Wolday D, Akuffo H, Petros B, Bronner $\mathrm{U}$, Britton S. Effect of deworming on human $\mathrm{T}$ cell responses to mycobacterial antigens in helminth-exposed individuals before and after bacille Calmette-Guérin (BCG) vaccination. Clin Exp Immunol. 2001;123(2):219-225.

26. Cunnington AJ, Riley EM. Suppression of vaccine responses by malaria: insignificant or overlooked? Expert Rev Vaccines. 2010;9(4):409-429.

27. Riner DK, et al. Schistosoma mansoni infection can jeopardize the duration of protective levels of antibody responses to immunizations against hepatitis B and tetanus toxoid. PLoS Negl Trop Dis. 2016;10(12):e0005180.

28. Stelekati E, Wherry EJ. Chronic bystander infections and immunity to unrelated antigens. Cell Host Microbe. 2012;12(4):458-469.

29. Schacker TW, et al. Collagen deposition in HIV-1 infected lymphatic tissues and T cell homeostasis. J Clin Invest. 2002;110 (8):1133-1139.

30. Kityo C, et al. Lymphoid tissue fibrosis is associated with impaired vaccine responses. J Clin Invest. 2018;128(7):2763-2773.

31. Utay NS, et al. Telmisartan does not improve lymph node or adipose tissue fibrosis more than continued ART alone. JInfect Dis. 2018;217(11):1770-1781. 